\title{
Constrained Inverse Volume Rendering for Planetary Nebulae
}

\author{
Marcus Magnor* \\ MPI Informatik, Germany
}

\author{
Gordon Kindlmann \\ SCI Institute \\ University of Utah, USA \\ Neb Duric \\ Dept. Physics and Astronomy \\ University of New Mexico, USA
}

\author{
Charles Hansen \\ SCI Institute \\ University of Utah, USA
}
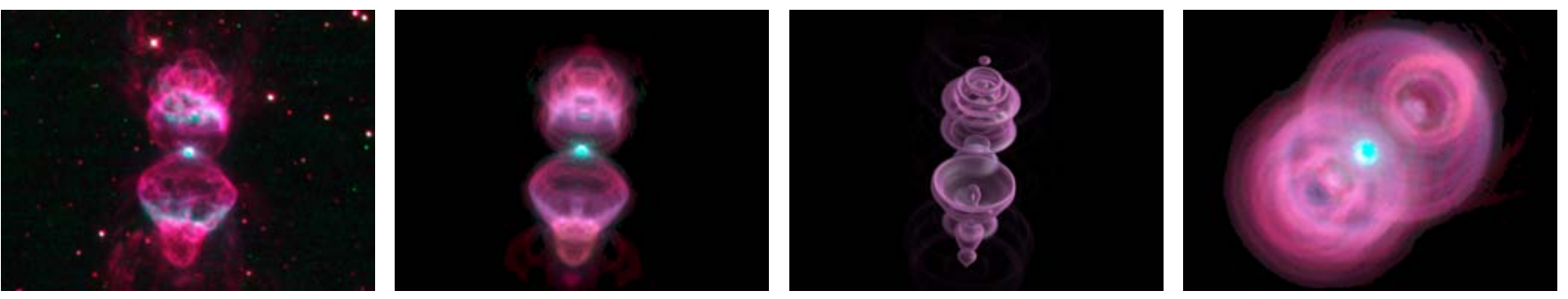

Figure 1: The planetary nebula Mz-3. Left to right: the nebula's color-composited original image, the same view rendered from the reconstructed volumetric model, a visualization of the nebula's iso-density surfaces, and a view of the nebula as seen from a vantage point in space thousands of light years from Earth.

\begin{abstract}
Determining the three-dimensional structure of distant astronomical objects is a challenging task, given that terrestrial observations provide only one viewpoint. For this task, bipolar planetary nebulae are interesting objects of study because of their pronounced axial symmetry due to fundamental physical processes. Making use of this symmetry constraint, we present a technique to automatically recover the axisymmetric structure of bipolar planetary nebulae from two-dimensional images. With GPU-based volume rendering driving a non-linear optimization, we estimate the nebula's local emission density as a function of its radial and axial coordinates, and we recover the orientation of the nebula relative to Earth. The optimization refines the nebula model and its orientation by minimizing the differences between the rendered image and the original astronomical image. The resulting model enables realistic 3D visualizations of planetary nebulae, e.g. for educational purposes in planetarium shows. In addition, the recovered spatial distribution of the emissive gas allows validating computer simulation results of the astrophysical formation processes of planetary nebulae.
\end{abstract}

CR Categories: I.3.m [Computer Graphics]: MiscellaneousConstrained Inverse Volume Rendering I.4.m [Image Processing and Computer Vision]: Miscellaneous-Volumetric Modeling; J.2

*e-mail: magnor@mpi-sb.mpg.de
[Computer Applications]: Physical Sciences and EngineeringAstronomy;

Keywords: volumetric modeling, inverse rendering, volume rendering, volume reconstruction, planetary nebulae

\section{Introduction}

Planetary nebulae $(\mathrm{PNe})^{1}$ are among the esthetically most appealing objects in the night sky, due to their diversity and colorful appearance. While it was soon realized that we are observing 2D projections of $3 \mathrm{D}$ volumes of glowing gas, the actual spatial structure of PNe has long been an object of inquiry. It was not until the second half of the 20th century that, based on earlier morphological classifications [Curtis 1918], qualitative models were proposed to explain the general appearance of PNe [Khromov and Kohoutek 1968; Balick 1987; Masson 1989]. While these simple models are able to qualitatively describe the great variety of observed PN shapes, they are not accurate enough to reflect the actual threedimensional structure of real planetary nebulae.

In this paper, we present a volume modeling technique coined constrained inverse volume rendering (CIVR) that recovers the actual structure of bipolar planetary nebulae from 2D images. Based on fast volume rendering using PC graphics hardware, we synthesize realistic views from a very general PN model. An optimization algorithm varies the model's emission distribution and compares the rendered model to the actual image until the rendered model matches the observed image. To make the problem well-posed, and at the same time obtain only physically plausible results, we constrain the reconstruction process by exploiting the axisymmetric nature of bipolar PNe, which is both physically derivable and observationally validated. The reconstructed axisymmetric emission

\footnotetext{
${ }^{1} \mathrm{We}$ will use "PN" and "PNe" to refer to planetary nebulae in the singular and plural, respectively.
} 
distribution enables creating realistic views of these interesting astronomical objects from arbitrary vantage points in space, e.g. for planetarium shows and educational purposes. Furthermore, as an astrophysical application, the recovered spatial distribution of emissive gas can serve in validating computer simulation results of PN formation processes.

In the following Section, we review previous work related to our contribution. After an introductory overview of the fundamental physics of PNe in Sect.3, we describe our constrained inverse volume rendering approach in Sect.4. Sect.5 illuminates the practical steps in reconstructing planetary nebulae. Results are presented in Sect.6. We discuss our method and its limitations as well as validate it in Sect.7, before we conclude in Sect.8 mentioning applications and giving an outlook on future improvements.

\section{Related Work}

The presented volumetric modeling technique relies on recent advances in visualization and graphics research. The term inverse volume rendering was first employed by Curless and Levoy [1996] to refer to updating volume data from range images. Mueller et al. [1998] use the same term to refer to a re-projection technique in CT reconstruction. Our use of the term is derived from Marschner's $\mathrm{Ph} . \mathrm{D}$. thesis [1998] in which he introduces the term inverse rendering to denote the idea of reconstructing scene properties from image data via computer graphics techniques.

The volume modeling approach we present is based on the ability to perform volume rendering [Levoy 1988] very efficiently on modern graphics hardware. Cabral et al. [1994] demonstrate how to perform efficient volume rendering using texture mapping hardware, a fundamental technique that is still applicable to today's programmable graphics architectures [Rezk-Salama et al. 2000]. For purely selfemitting volumes, Cabral's slicing technique directly yields correct rendering results [Max 1995].

While spectacular 3D fly-throughs of astronomical objects can be experienced in many planetariums, these animations are almost always purely artistic pieces of work. One notable exception is the effort by Nadeau et al. [2001] who used massive computational power to create scientifically accurate views of the Orion nebula for the Hayden Planetarium in New York [Genetti 2002]. For their visualizations, they rely on a 3D model of the Orion nebula that was determined by astronomers from decades of observational data [Zheng and O'Dell 1995]. Our method, in contrast, simultaneously reconstructs the 3D volume model and visualizes it, albeit for a different class of astronomical objects: planetary nebulae.

\section{Planetary Nebulae}

In the following, we give a brief explanatory overview of the main physical processes that lead to the visual appearance of PNe. For a more thorough introduction to the physics of gaseous nebulae in general and PNe in specific, we recommend the classic books on the topic by Osterbrock [1989] and Kwok [2000].

Despite their name, planetary nebulae are of extra-solar origin: they constitute the last episode in the lives of most stars. On astronomical scales, they are extremely short-lived, existing only for a few tens of thousands of years before they fade away, which is why only about 1,500 have been discovered in our Galaxy so far. Owing to their great distances from earth, they are small, spanning at most a few arc minutes in the sky, and dim. For the work presented here,

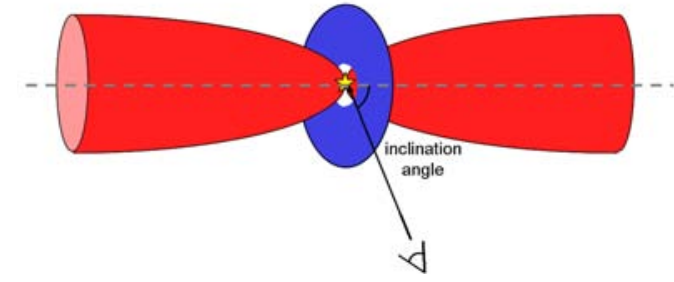

Figure 2: Interacting winds theory: During its lifetime, a torus of dense, slowly outward moving gas gathers in the equatorial plane of the central star (blue). At the end of its life, the star suddenly blows off gas at a much higher rate. When this fast wind hits the inner boundary of the old gas masses, the fast-moving gas is re-directed and escapes in opposite, polar directions (red).

we rely on publicly available images that have been recorded with the Hubble Space Telescope [Hubble 2004] and the Nordic Optical Telescope at Teneriffe, Canary Islands [IAC 2004].

\subsection{Photo-ionization}

When a star of initially less than eight times the mass of the Sun nears its end of energy production, a strong wind blows off its surface, carrying substantial amounts of stellar matter into space and exposing deeper, hotter regions of the star. This wind consists mainly of hydrogen but also of other elements that were previously synthesized within the star's core. At the same time, the central star contracts which increases the exposed surface's temperature even further. Following Wien's law for blackbody radiation [Osterbrock 1989], with rising surface temperature the star emits more high-energy ultra-violet (UV) photons. Once the star emits enough UV photons carrying sufficient energy to ionize hydrogen $(13.6 \mathrm{eV}$, corresponding to a wavelength of $91 \mathrm{~nm}$ ), the ejected hydrogen gas becomes completely photo-ionized, i.e. essentially all hydrogen atoms are being separated into free electrons and hydrogen ions (protons). Within this plasma, both constituents frequently recombine to form neutral hydrogen atoms again. These newly formed atoms are typically in an excited state, however, from which they return to the ground state via a cascade of energy transitions, before another UV photon ionizes the atom again. Each transition during this cascade is accompanied by the emission of a photon of a distinct wavelength. These emission-line photons can escape from the nebula essentially unhindered, and it is these photons that we observe and that are responsible for the nebula's characteristic color.

Besides hydrogen, the other chemical elements in the nebula are also in ionized states and emit photons at characteristic wavelengths. Thus, images recorded at different wavelengths enable astronomers to identify different elements in different regions of a nebula, Fig.4. For most (mature) PNe, the central star's UV photon flux is more than sufficient to photo-ionize all gas that was previously blown off the star. Assuming that the amount of lightabsorbing dust can be neglected, we observe the entire mass of ejected gas as one glowing volume in space [Kwok 2000].

\subsection{Axisymmetry}

Planetary nebulae glow because gas previously ejected from the central star is being ionized by the star's intense UV radiation. Photo-ionization alone, however, cannot explain the axial symmetry of bipolar PNe, since the central star certainly radiates isotrop- 
ically into all directions. Instead, it is the non-uniform spatial distribution of the gas that is responsible for the bipolar appearance of many $\mathrm{PNe}$.

A classification scheme for PNe based on visual morphology was proposed by Curtis early in the 20th century [1918]. In the 1960s, it became clear that the various different shapes of PNe may be qualitatively explained by one common, general shape viewed from Earth at different orientations [Khromov and Kohoutek 1968]. While this phenomenological model was motivated by observational data, it did not explain the physical processes leading to the shape. Kwok [1978] and Balick [1987] finally proposed a mechanism that explains the observed gas distribution in PNe, Fig.2; their interacting winds theory starts with the premise that prior to its final stage, the star has already been blowing off gas, but at a much slower speed. Due to the star's rotation and the resulting centripetal force, this wind was slightly stronger along the equator, and the ejected gas accumulated in the equatorial plane. As the rate of gas discharge increases towards the end of the star's life, the faster and stronger wind collides with the old gas masses along the star's equator. A shock front develops, and the fast outflowing gas is redirected away from the equator, towards the poles. Two lobes of gas arise, flowing away from the central star into opposite directions. This phenomenon is amplified in the presence of a magnetic field from the central star which focuses the outflow of the photoionized gas plasma. Additionally, the fast wind might already be blowing off stronger from the polar regions. From the symmetry of the system and the conservation of momentum, the outflowing gas jets cannot do anything else but be axisymmetric, creating the bipolar shape of many PNe [Morrisset et al. 2000].

In extensive gas-dynamic [Mellema and Frank 1989] and photoionization simulations [Morrisset et al. 2000; Ercolano et al. 2003] the plausibility of the interactive winds theory has been demonstrated. On its basis, a low-dimensional (4 degrees of freedom) shape model for PNe, the prolate ellipsoidal shell (PES) model, was proposed by Masson [1989]. The PES model was subsequently used by Kwok et al. [1996; 1998] to model the general appearances of a number of PNe by adjusting the four model parameters by hand. However, its simple geometry can only give a rough, qualitative account of the spatial density distribution of the gas within PNe.

\section{Constrained Inverse Volume Rendering}

The nature of bipolar PNe enables us to develop a method for automatic reconstruction of the axisymmetric emissive gas distribution from two-dimensional images as observed from Earth. Our constrained inverse volume rendering (CIVR) approach thereby relies on three key physical and observational properties:

- Bipolar PNe exhibit a symmetry axis. This axisymmetry reduces the 3D nebula volume to a 2D emission density function in cylindrical coordinates.

- Scattering and absorption is assumed to be negligible at the visible wavelengths at which our astronomical input images are acquired.

- Planetary nebulae span only a few arc minutes in the sky (less than a tenth of a degree), so their projected appearance is essentially orthographic.

Our model consists of a two-dimensional density map whose entries represent axisymmetric emission density values. The map is aligned with the symmetry axis of the nebula such that the central star is in the middle, Fig.3. The CIVR algorithm determines all map

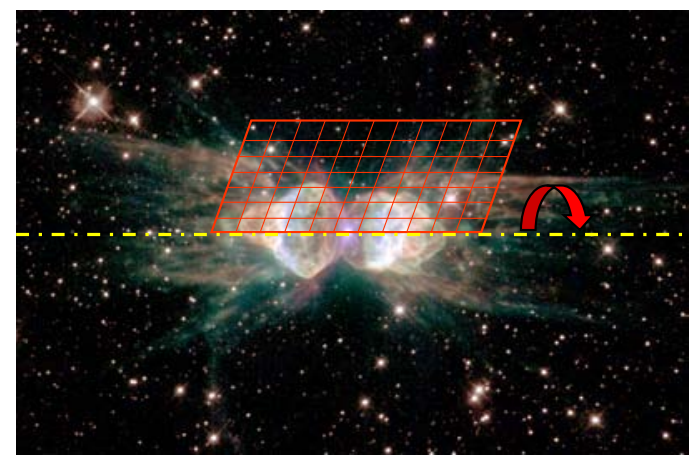

Figure 3: Axial symmetry: the structure of bipolar planetary nebulae can be described by a density map in cylindrical coordinates. The volumetric density distribution in 3D space is generated by rotating the density map along the nebula's axis of symmetry.

entry values such that the corresponding projection image, obtained by rotating the emission density map around the symmetry axis and integrating emission density along the line of sight, matches the nebula's photograph.

Unfortunately, the relationship between the axisymmetric density map and the nebula's observed projection image remains nonlinear, since we do not know the nebula's 3D orientation. Also, map entries can only have non-negative values. We therefore pursue a non-linear optimization approach that relies on repeatedly evaluating an error function that measures the difference between the projected PN model and the photo of its celestial counterpart. Thanks to fast volume rendering on modern graphics hardware, this error function can be evaluated very efficiently.

\subsection{Emissive Volume Rendering}

Since PNe consist of glowing gas, synthesizing faithful projection images from our PN model consists of rendering a purely emissive volume. Such straight-forward volume rendering can be implemented very efficiently on PC graphics hardware by rendering multiple viewport-aligned slices through the nebula volume at different depths and summing up all rendered slices [Cabral et al. 1994]. For each depth layer, a quadrilateral perpendicular to the viewing direction and spanning the entire viewport is sent through the graphics pipeline. During rasterization, a small fragment program transforms the current rasterization coordinates to cylinder coordinates and performs the bilinearly interpolated look-up into a 2D texture map that represents the axisymmetric emission density map. To add up all rendered slices, we make use of the floating point buffer available on modern graphics hardware. An nVidia GeForce FX 3000 graphics card easily attains $10 \mathrm{fps}$ when rendering 128 depth layers at $128 \times 128$ image resolution.

\subsection{Projection Error Evaluation}

The aim of CIVR is to determine the emission density map entries such that its $2 \mathrm{D}$ projection, the volume rendering result, matches the true PN image as closely as possible. This is accomplished by comparing the rendering result to the original PN image and systematically varying the emission density map values until the projection error is minimal.

To quantify the difference between the model projection and the true image, the floating point buffer is read to local memory. Each 
rendered pixel is compared to its corresponding pixel in the PN image, and the sum-of-squared-differences over all pixels is computed. An optimization routine then varies the density map entries and repeatedly calls the volume rendering algorithm to determine the corresponding projection error. Since emission density cannot become negative, a very high error value is returned whenever the optimization routine attempts to set a map entry to a negative value. This allows the otherwise generic optimization routine to be constrained to a physically plausible PN model.

\subsection{Optimization}

We employ a standard implementation of Powell's optimization method [Press et al. 1992] to find the best emission density map entries. It numerically finds the error function's local slope along all dimensions from which it determines the conjugate-gradient direction. Each error function evaluation entails modifying the density map entries and orientation angle values, uploading the new map values to the graphics card, volume-rendering the model, and calculating the sum-of-squared-differences measure. This analysisby-synthesis loop iterates until the algorithm converges to a minimum of the error function. The optimization benefits from employing a multi-resolution strategy, in which coarse-resolution model versions are optimized first which are subsequently used for initialization of the next higher-resolution model level, Sect.5.2.

\section{Reconstruction}

For PN reconstruction, the CIVR algorithm is applied to images of planetary nebulae recorded at optical wavelengths. Mathematically, the recovery of the axisymmetric emission density distribution from a 2D image is equivalent to de-projecting the image, i.e. a deconvolution with a point spread function that depends on the (unknown) $3 \mathrm{D}$ orientation of the nebula. This de-projection becomes possible thanks to the nebula's axial symmetry if the symmetry axis is oriented roughly perpendicular to our viewing direction.

\subsection{Pre-processing}

The constrained inverse volume rendering method takes optical images of planetary nebulae as input. Research-grade images of many PNe are publicly available on the Internet. The data used for our work, and the astronomical images shown here, are from the Hubble Space Telescope Archive [Hubble 2004] and the IAC Morphological Catalog of Northern Galactic Planetary Nebulae [IAC 2004]. The images have been acquired with narrow-bandpass filters at distinct wavelengths that correspond to specific chemical elements, Tab.2. The scalar-valued images exhibit a reliable linear dynamic range of about 16 bits.

Prior to applying our CIVR volume modeling method, the images must be pre-processed to correct for a number of imaging-related effects in order to obtain scientifically accurate data. For our CIVR technique to produce correct results, we need images that are calibrated to exhibit a linear response curve, i.e. twice the number of photons falling on a pixel must double the pixel's value. We calibrate the raw image data using the standard astronomical image processing software IRAF [IRAF 2004] and STSDAS [STSDAS 2004]. Stars nearby and behind the nebula interfere with the reconstruction process because their light originates outside the nebula model. We manually mask out such regions. To avoid bias in the reconstruction result, the CIVR algorithm disregards these regions when computing the error function; the masked pixels are effectively missing from the image. Due to imaging noise, the background pixels in the images additionally exhibit small, non-zero values. This is a second potential source of inaccuracy since the CIVR algorithm would attempt to model the pixels outside the nebula as part of the nebula itself. Thus, we threshold the images and set the background uniformly to zero. Finally, the PN's central star is identified in each image, and the nebula's projected axis of symmetry is approximately determined. This can be done automatically by finding the maximum pixel value in the image (corresponding to the central star's position) and calculating the principal eigenvectors of the image's covariance matrix.

PNe are small objects in the sky, and atmospheric turbulence typically limits useful imaging resolution from ground-based telescopes to a couple of hundred pixels along the longest dimensions. To determine at which resolution our axisymmetric density map may be optimally reconstructed, we rely on the Nyquist theorem, stating that the highest measurable frequency corresponds to 2 samples per period. Then, for a nebula covering 256 pixels in the image along its longest direction, we can sensibly expect to recover 128 pixels in our density map. Finally, the PN image is centered on the central star and clipped to $256 \times 256$ pixels, which is sufficiently large to completely accommodate the nebulae in all our test images.

\subsection{Volumetric Modeling}

We now apply our CIVR algorithm to each calibrated grey-scale image individually. Initially, all density map entries are set to zero. Because the nebula's symmetry axis goes through the central star, we initialize one orientation angle based on the slope of the PN's line of symmetry in the image. The second, unknown angle, representing the inclination of the nebula's symmetry axis towards Earth, is initialized to be perpendicular to our viewing direction. During CIVR, both angles are also optimized in addition to the emission density map entries in order to determine the true $3 \mathrm{D}$ orientation of the nebula.

To accelerate convergence, our CIVR implementation follows a multi-resolution approach. Reconstruction starts from a lowresolution version of the density map and the image. At this level, only a fraction of density map entries must be reconstructed, and the model is volume-rendered very quickly at low resolution. After convergence, the reconstructed low-resolution density map is scaled up to serve as initialization for the next-higher resolution density map. When doubling the map's resolution, rendering resolution must be increased likewise, which in turn increases rendering times during error function evaluation. Employing four hierarchy levels, the reconstruction of a 128x32-pixel density map takes approximately one day on a $2.4 \mathrm{GHz} \mathrm{PC}$ in conjunction with an nVidia GeForce FX 3000 graphics card.

\begin{tabular}{lll} 
PN & incl. angle & orient. angle \\
\hline He2-437 & $84^{\circ} / 96^{\circ}$ & $77.5^{\circ}$ \\
M1-92 & $79^{\circ} / 101^{\circ}$ & $16.5^{\circ}$ \\
Mz-3 & $55^{\circ} / 125^{\circ}$ & $175.5^{\circ}$
\end{tabular}

Table 1: Reconstructed nebula orientations. The inclination angle can only be determined up to a symmetry ambiguity with respect to the perpendicular $90^{\circ}$ orientation. 

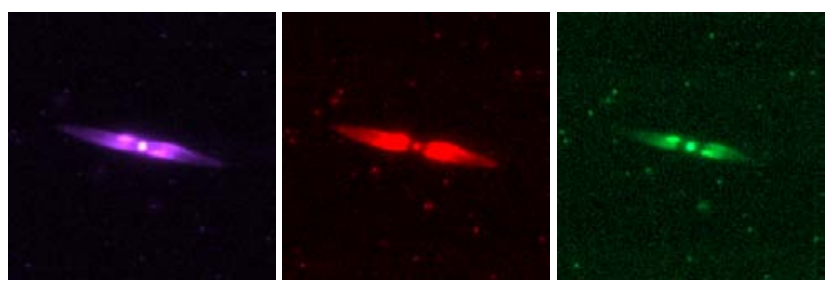

Figure 4: Input images: The planetary nebula He2-437 in the light of hydrogen, nitrogen and oxygen (from left to right). While the original image data is scalar-valued (grey-scale images), correct color appearance is determined by taking into account the complete emission line spectrum of each chemical element (Sect.6.1).

\section{Results}

We evaluated our CIVR algorithm using images from three different PNe. For each planetary nebula, we have available several images taken at different wavelengths, corresponding to different chemical elements, Fig.4. Separate axisymmetric density maps have been reconstructed, depicting the elements' spatial abundances throughout the nebula, Fig.5. By rotating these axial density maps along their upper edge, the nebula's three-dimensional emission density distribution is constructed. Figs.1,6 depict color composites of the actual PN images alongside with renderings from the same viewpoint obtained from the reconstructed models and visualizations of iso-density surfaces within the nebulae. The volume renderings in the third column use multiple soft isosurfaces of the gas density to help convey the 3D structure of the PNe. The coloring on the isosurface originates from a volume RGB texture generated similarly to the images described in the next Section. Diffuse shading aids in interpreting 3D shape. In addition to the emission density maps, the orientation angles of each nebula are determined, Tab.1. Note that the orthographic projection property implies that the inclination angle can only be recovered up to a two-fold ambiguity, yielding symmetrical solutions with respect to the perpendicular orientation. By assuming an equatorial dust belt around the central star, this symmetry can be broken, however, and the dimmer of either lobe must be behind the absorbing dust, as visible for M1-92 in Fig.6.

\subsection{Realistic 3D Visualization}

Our reconstructed PN models enable us to accurately visualize these attractive objects from arbitrary viewpoints, useful e.g. for planetarium shows and other educational purposes. To achieve the most natural visual impression, we wish to render the nebulae in accurate colors.

For the depicted nebulae, we made an effort to reproduce their actual colors. For each PN, we have available images corresponding to one characteristic emission line for the elements hydrogen, oxygen, and nitrogen or sulfur, Fig.4. These are the elements responsible for the visible color appearance of PNe. We assume that the images have been obtained using narrow-band filters which single out only the respective element's emission line. Tab. 2 depicts all relevant emission lines in the visible region of the spectrum and their relative intensities. While relative emission line strengths are somewhat dependent on the physical conditions inside the nebula (temperature, electron density), we have adopted here mean values for PNe as published in the literature [Osterbrock 1989; Kwok 2000]. Having rendered a view using all elements' emission density maps, for each pixel we construct its spectrum according to the ele-

\begin{tabular}{lcc} 
element & wavelength & rel. line strength \\
\hline \multirow{3}{*}{ hydrogen } & $656 \mathrm{~nm}$ & 1.00 \\
& $486 \mathrm{~nm}$ & 0.35 \\
& $434 \mathrm{~nm}$ & 0.16 \\
& $410 \mathrm{~nm}$ & 0.09 \\
oxygen & $501 \mathrm{~nm}$ & 1.00 \\
& $496 \mathrm{~nm}$ & 0.33 \\
nitrogen & $436 \mathrm{~nm}$ & 0.01 \\
& $658 \mathrm{~nm}$ & 1.00 \\
& $655 \mathrm{~nm}$ & 0.33 \\
sulfur & $575 \mathrm{~nm}$ & 0.02 \\
& $673 \mathrm{~nm}$ & 1.00 \\
& $672 \mathrm{~nm}$ & 0.6
\end{tabular}

Table 2: Nebula colors: Planetary nebulae owe their colorful appearance to a handful of discrete emission lines. The table depicts emission-line wavelengths for the relevant elements and their relative intensities (last column). By imaging the nebula at one wavelength for each element, the intensities of the other emission lines can be deduced, and the nebula can be rendered in correct color.

ment emissions' intensities, Tab.2, which we convert to RGB values using the CIE 1931 XYZ color matching function values and the appropriate color space transformation [Wyszecki and Stiles 1982].

Planetary nebula images exhibit a wide dynamic range. While the central star typically represents the brightest region in any PN image, the outermost regions of glowing gas frequently drown in detector noise. To simultaneously display the bright regions and the faint structure of the gas, we employ simple gamma correction to all three color channels. More advanced tone mapping operators may yield visually even more attractive rendering results [Reinhard et al. 2002]. Fig.7 depicts our planetary nebulae as seen under different inclination angles, corresponding to viewpoints located thousands of light years from Earth [Videos ]. Notice the considerable change in appearance with orientation angle, but also the diversity among different planetary nebulae. By studying the variety in possible PN appearance, the CIVR results may help astronomers identify planetary nebulae seen at low inclination angles that have so far eluded identification.

\subsection{Interpretation}

An astrophysical interpretation of the reconstructed axisymmetric maps must be done with care. Especially our negligence to absorption in and around the nebula can lead to systematic errors. This is obvious for M1-92, Fig.6 whose southern lobe appears much darker than the northern lobe, which is probably due to an equatorial belt of absorbing dust around the central star. It is nevertheless noticeable that emission density varies considerably within each nebula, indicating that the gas is distributed rather inhomogeneously. In addition, concentration maxima of different chemical elements appear to be located in different regions. Finally, spatial distribution differs substantially between different PNe. In He2-437, large regions of empty space are present within the nebula. M1-92, in contrast, features two gas lobes with small, localized density concentrations along the axis of symmetry. The distribution in Mz-3 is even more complex, with most emission being concentrated along the shock front. 


\section{Discussion}

The CIVR method reconstructs the axisymmetric structure of $\mathrm{PNe}$ such that the re-projection is a very close match to the actual, recorded image. In the following, we validate the credibility of CIVR results and discuss the inherent limitations of our reconstruction approach.

Since our reconstruction problem is non-linear, a proof for general convergence to the global minimum of our error function cannot be given. To validate the reconstruction results derived for real nebulae, we would have to know the actual 3D distribution. While there exists a rather involved, indirect observation technique known as echellogrametry [Sabbadin 1984; Sabbadin et al. 2000] to reconstruct 3D gas velocities in spherical nebulae, no measured 3D gas distribution data is available for bipolar planetary nebulae. Instead, we must rely on synthetic images to test our approach. We make use of Aaquist and Kwok's ellipsoid shell model [1996] to generate test images for a number of different orientations. We then let our CIVR algorithm recover the axisymmetric density distribution and orientation angles and compare the reconstructed values with the original values derived from the ellipsoid shell model. For inclination angles down to 40 degrees, the reconstructed map values are within the error range that can be expected from the optimization routine's preset termination error threshold. The accuracy of the recovered inclination angle varies with angle value, from about $3^{\circ}$ error for 90-degree inclination down to less than half a degree at $45^{\circ}$ inclination. While we cannot test all possible cases, our validation experiments indicate that CIVR reconstruction results do, indeed, closely resemble the true 3D structure of planetary nebulae.

CIVR can be applied not only to bipolar PNe but also to spherical nebulae by exploiting their point symmetry. Only for irregularly shaped nebulae, e.g. if the outflowing gas collides with interstellar gas clouds, our reconstruction approach is not applicable. Our method works best for nebulae seen at high inclination angles, i.e. for PNe whose axis of symmetry is oriented approximately perpendicular to our viewing direction. This can be understood by approximating the reconstruction as an inverse linear problem and looking at the condition number of the projection matrix. With decreasing inclination angle, i.e. when the PN's symmetry axis approaches the viewing direction, the condition number increases and the (unregularized) reconstruction result becomes unstable. At $0^{\circ}$ inclination, the symmetry axis points directly towards the observer, and the nebula's axial symmetry is of no use to constrain the reconstruction problem: the linear system of equations is under-determined. Our validation experiments indicate that CIVR is capable of robustly reconstructing $\mathrm{PNe}$ down to inclination angles of $40^{\circ}$.

\section{Conclusions}

We have presented a constrained inverse volume rendering (CIVR) approach to recover the three-dimensional distribution of the photoionized gas in bipolar planetary nebulae $(\mathrm{PNe})$ from optical images. Thanks to their inherent axial symmetry, we are able to use an axisymmetric model to describe the nebula's emission distribution in space, thereby constraining the reconstruction problem. Being purely emissive, the PN model is volume-rendered efficiently using modern PC graphics hardware. By comparing the rendering result with the actual image, an optimization algorithm varies local emission as well as orientation angles to match the rendered model to the recorded image.

From the reconstructed spatial emission distribution, the nebulae can be rendered from arbitrary perspective. Applications include creating realistic visualizations of $\mathrm{PNe}$ as illustration material for astronomy courses, science programs, and planetarium shows. Collaborations with a number of planetariums are underway. Furthermore, the recovered axisymmetric shape is useful for studying the spatial relationships of element abundances, as well as to validate and refine the interacting winds theory. By comparing CIVR reconstruction results to photo-ionization and gas-dynamic simulations [Armsdorfer et al. 2000], astrophysical models of planetary nebulae can be validated. This way, a general evolutionary sequence among observed PNe might be established that can explain the variation in composition and appearance based on only a handful of physical properties of the central star.

Next, we intend to take into account also the dust component during reconstruction. Extinction maps can be reconstructed from additional radio-continuum observations which are not affected by dust absorption. By making the plausible assumption that the dust distribution also exhibits axial symmetry, the spatial distribution of the ionized gas and the dust can be recovered simultaneously. As a beneficial side effect, the reconstructed dust distribution will resolve the two-fold ambiguity in the recovered inclination angle. For reconstruction, we have not made use of regularization and did not enforce smoothness in our reconstructed density maps which can be easily incorporated into our energy function. For noisy images, or if the nebula is only approximately axisymmetric, such additional constraints will stabilize reconstruction. Another intriguing extension yielding visually even more attractive results consists of reconstructing the 3D structure in two steps; after the general axisymmetric structure has been recovered, the remaining differences between the rendered model and the image may be attributed to emission density variations on the nebula's shock front, i.e. where the expanding gas collides with the interstellar medium. This assumption is plausible since small-scale density variations are most probably due to turbulence along the fast stellar wind's shock front. This way, non-axisymmetric details present in PN images are incorporated into the model and can be replicated during visualization.

\section{Acknowledgments}

We wish to express our gratitude to Sun Kwok at the University of Calgary, Canada, and to Stefan Kimeswenger at the University of Innsbruck, Austria, two experts on planetary nebulae, for their helpful comments and their feedback on the usefulness of the presented method for astrophysics research. Thanks also to Bastian Goldlücke for his help in programming around the graphics hardware driver's bugs, and to Andrei Lintu for calibrating the astronomical images. This project was funded in part by the German Science Foundation DFG.

\section{References}

Aaquist, O., AND KWoK, S. 1996. Radio morphologies of planetary nebulae. Astrophysical Journal 462, 2 (May), 813-824.

Armsdorfer, B., Kimeswenger, S., And Rauch, T. 2000. Effects of Central Star Models on Planetary Nebulae Shell Modelling. In Ionized Gaseous Nebulae. Mexico City November 21- 24, 2000.

BALICK, B. 1987. The evolution of planetary nebulae. I - structures, ionizations, and morphological sequences. Astronomical Journal 94 (Sept.), 671-678.

CABral, B., CAM, N., AND Foran, J. 1994. Accelerated volume rendering and tomographic reconstruction using texture mapping hardware. Proc. Symposium on Volume Visualization (VolVis'94), 91-98.

Curless, B., AND Levoy, M. 1996. A volumetric method for building complex models from range images. Proc. ACM SIGGRAPH (Computer Graphics Series) $30,303-312$. 

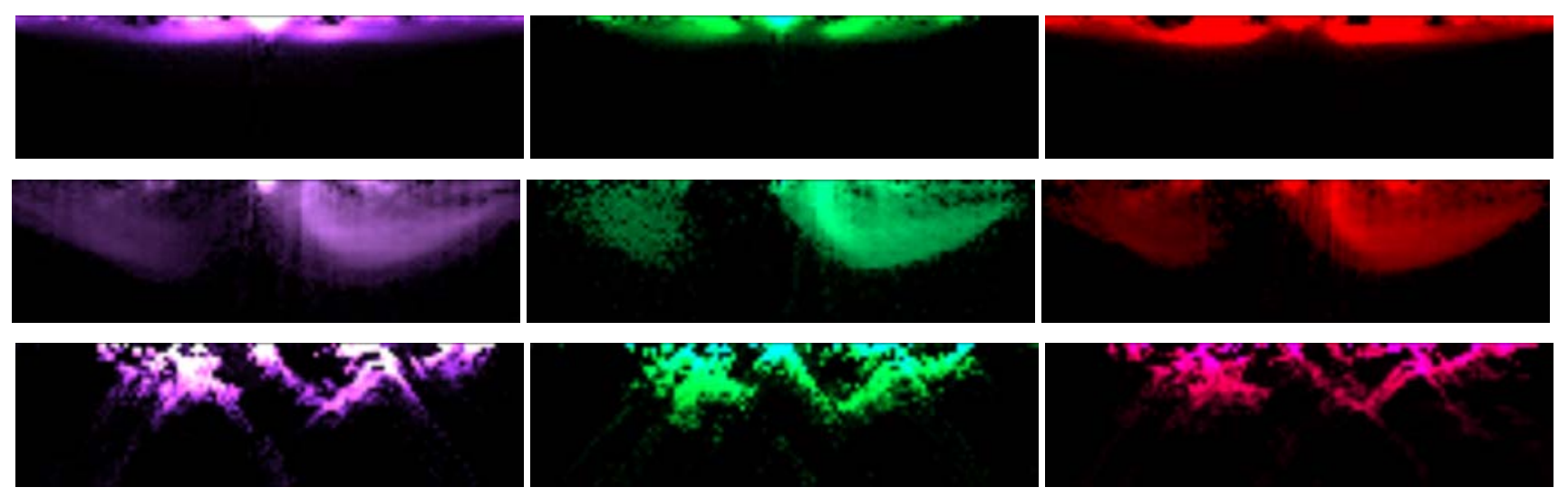

Figure 5: Axisymmetric structure of the planetary nebulae He2-437, M1-92, and Mz-3 (top to bottom). From left to right, the maps illustrate the distribution of the elements hydrogen, oxygen, and nitrogen (sulfur for Mz-3).

CurTIS, H. 1918. The planetary nebulae. Publ. Lick Observatory, part III, 13, 57-74.

Ercolano, C., Morisset, C., Barlow, M., Storey, P., And Liu, X.-W. 2003. Three-dimensional photoionization modelling of the planetary nebula NGC 3918. Monthly Notices of the Royal Astronomical Society 340, 1153-1172.

Genetti, J. 2002. Volume-rendered galactic animations. Communications of the ACM 45, 11 (Nov.), 62-66.

HuBBLE, 2004. http://archive.stsci.edu/hst/search.php.

IAC, 2004. http://www.iac.es/nebu/nebula.html.

IRAF, 2004. http://iraf.tuc.noao.edu.

Khromov, G., And Kohoutek, L. 1968. Morphological study of planetary nebulae. In Planetary Nebulae, D. Osterbrock and C. O'Dell, Eds., IAU Symposium 34, 227-235.

Kwok, S., Purton, C., And Fitzgerald, P. 1978. On the origin of planetary nebulae. Astrophysical Journal 219, L125-L127.

KwoK, S. 2000. The Origin and Evolution of Planetary Nebulae. Cambridge University Press, Cambridge, UK.

LEvoY, M. 1988. Display of surfaces from volume data. IEEE Computer Graphics and Applications 8, 5, 29-37.

MARSCHNeR, S. 1998. Inverse Rendering for Computer Graphics. $\mathrm{PhD}$ thesis, Cornell University.

MASson, C. 1989. The structure of and distances to BD + 30 deg 3639 and NGC 6572. Astrophysical Journal 346, 243-250.

MAX, N. 1995. Optical models for direct volume rendering. IEEE Trans. Visualization and Computer Graphics 1, 2, 99-108.

Mellema, C., AND Frank, A. 1989. Radiation gasdynamics of planetary nebulae V. hot bubble and slow wind dynamics. Monthly Notices of the Royal Astronomical Society 273, 401-410.

Morrisset, C., Gruenwald, R., And Viegas, S. 2000. Morphology and kinematics of planetary nebulae. I a new modeling tool. Astrophysical Journal 533 (Apr.), 931-937.

Mueller, K., YAGel, R., AND Wheller, J. 1998. A fast and accurate projection algorithm for the algebraic reconstruction technique (ART). SPIE Medical Imaging Conference, San Diego, USA SPIE 3336 (Feb.), 724-732.

Nadeau, D., Genetti, J., Napear, S., Pailthorpe, B., Emmart, C., WesSELAK, E., AND DAVIDSON, D. 2001. Visualizing stars and emission nebulae. Computer Graphics Forum 20, 1 (Mar.), 27-33.

OSTERBRock, D. 1989. Astrophysics of Gaseous Nebulae and Active Galactic Nuclei. University Science Books, Sausalito, CA.

Press, W., Flannery, B., Teukolsky, S., And Vetterling, W. 1992. Numerical Recipes in C. Cambridge University Press.

Reinhard, E., Stark, M., Shirley, P., And Ferwerda, J. 2002. Photographic tone reproduction for digital images. ACM Trans. Graphics 21, 3, 267-276.
Rezk-Salama, C., Engel, K., Bauer, M., Greiner, G., and Ertl, T. 2000. Interactive volume rendering on standard $P C$ graphics hardware using multitextures and multi-stage rasterization. Proc. ACM SIGGRAPH/EUROGRAPHICS Workshop on Graphics Hardware, 109-118.

Sabbadin, F., Cappellaro, E., Benetti, S., Turatto, M., and Zanin, C. 2000. Tomography of the low excitation planetary nebula NGC 40. Astronomy \& Astrophysics 355 (Mar.), 688-698.

SABBADIN, F. 1984. Spatiokinematic models of five planetary nebulae. Monthly Notices of the Royal Astronomical Society 210, 341-358.

SAURER, W. 1997. Morphology and expansion characteristics of the planetary nebula M 1-79. Astronomy \& Astrophysics 326 (Oct.), 1187-1194.

Schwarz, H. E., And Monteiro, H. 2003. Properties of Bipolar Planetary Nebulae. In Revista Mexicana de Astronomia y Astrofisica Conference Series, 23-28.

Schwarz, H. E., Aspin, C., Corradi, R. L. M., And Reipurth, B. 1997. M 2-9: moving dust in a fast bipolar outflow. Astronomy Astrophysics 319 (Mar.), 267-273.

STSDAS, 2004. www.stsci.edu/resources/software_hardware/stsdas.

VIDEOS. http://www.mpi-sb.mpg.de/rmagnor/civr.html.

WysZeCKi, G., AND Stiles, W. S. 1982. Color Science: Concepts and Methods, Quantitative Data and Formulae, 2nd ed. John Wiley \& Sons, New York.

ZHANG, C., AND KWOK, S. 1998. A morphology study of planetary nebulae. Astrophysical Journal Supplement Series 117, 2 (Aug.), 341-359.

ZhenG, W., AND O'DELL, C. 1995. A three-dimensional model of the Orion nebula. Astrophysical Journal 438, 2 (Jan.), 784-793. 


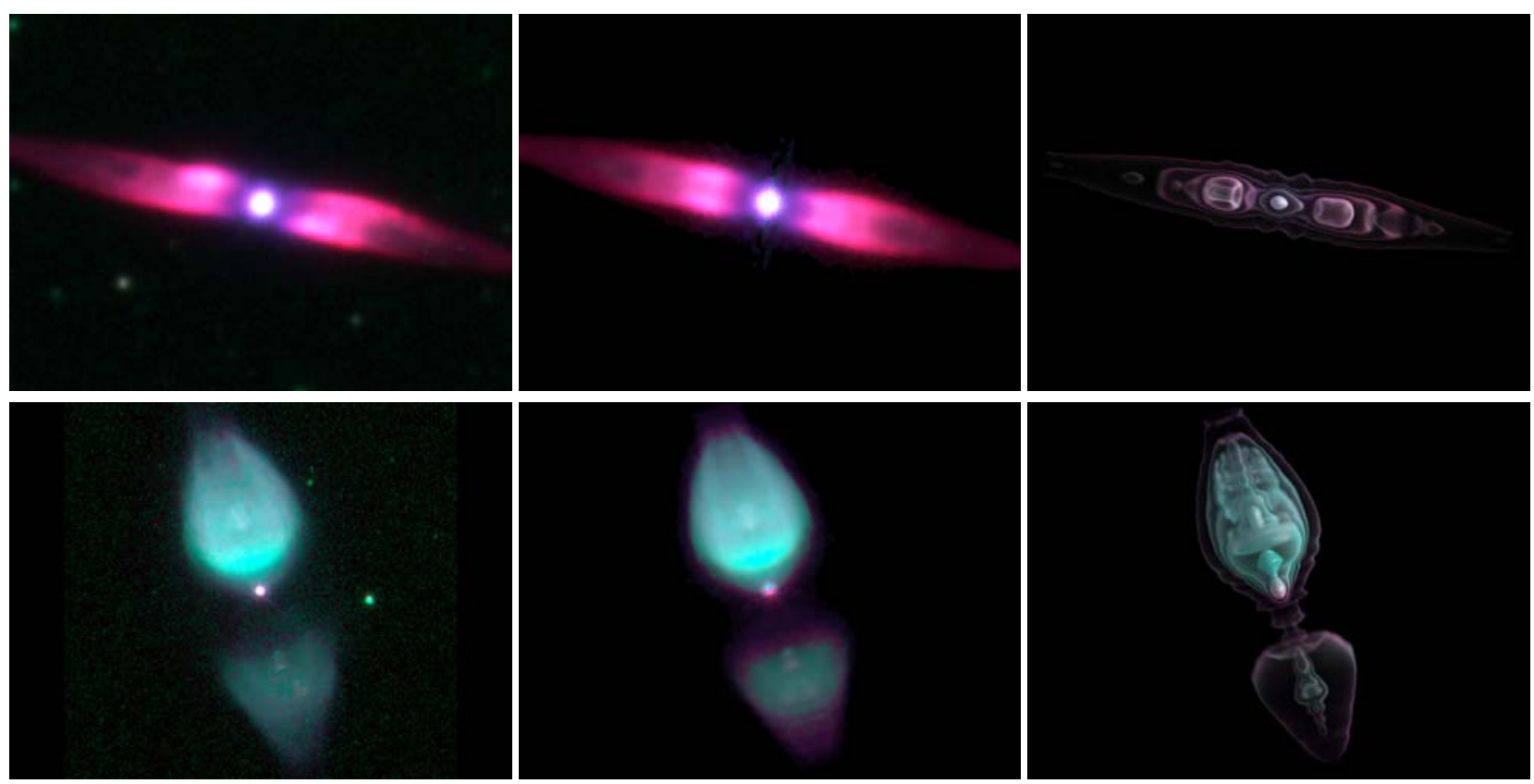

Figure 6: CIVR modeling results for the planetary nebulae He2-437 (top) and M1-92 (bottom). Left to right: the color-composited original image, the same view rendered from the reconstructed model, and a visualization of the nebula's iso-density surfaces.

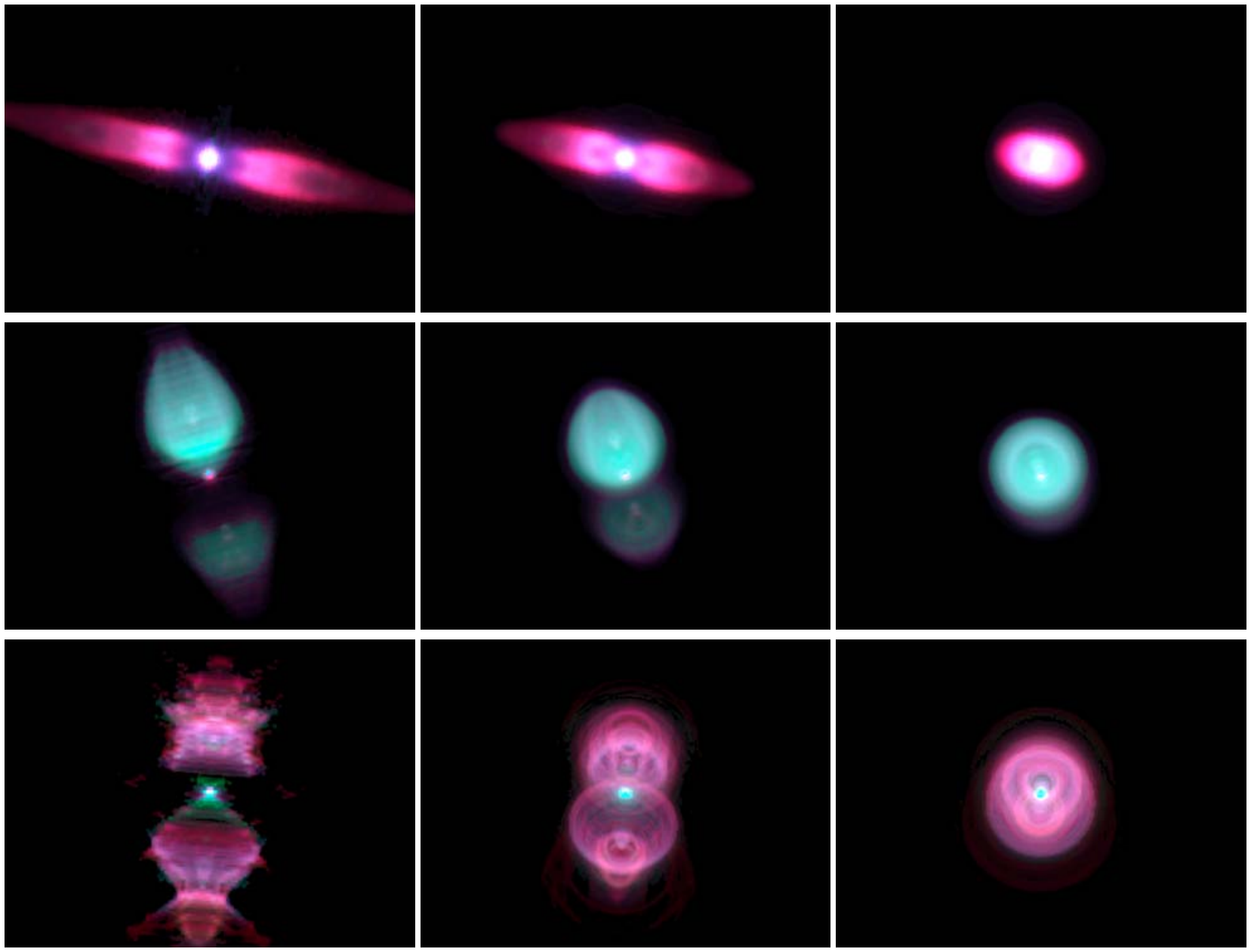

Figure 7: Unfamiliar sights: He2-437, M1-92, and Mz-3 (top to bottom) at inclination angles $85^{\circ}, 35^{\circ}$, and $10^{\circ}$ (left to right). 